\title{
The role of the brain's frontal eye fields in constructing frame of reference
}

\author{
Mikkel Wallentin
}

Published online: 18 July 2012

(C) Marta Olivetti Belardinelli and Springer-Verlag 2012

\begin{abstract}
Establishing contextual reference during discourse is a vital part of language function. Personal pronouns (e.g., he/she/it) are used to refer to previously experienced objects, utterances and events. These items, however, are often no longer present in the environment and have to be maintained and manipulated in working memory (WM). One aspect of this is the construction of a spatial frame of reference (e.g., "He was in front of it" where "he" is established as figure and "it" is the ground). The WM processes underlying this function may be different from those involved in establishing a non-spatial relation (e.g., "He was older than her"). The brain's frontal eye fields (FEFs), responsible for eye movement control, are known to be involved in processing spatial WM. This paper reviews both functional magnetic resonance imaging experiments and a subsequent behavioral interference study demonstrating a specific role for the FEFs and the brain's eye movement control system in manipulation of WM content for establishing object-centered spatial reference frames during verbally cued recall of recent visual and linguistic experiences.
\end{abstract}

Keywords Spatial perspective - Working memory · Frontal eye fields $\cdot$ Interference task

M. Wallentin

Center for Semiotics, Aarhus University, Aarhus, Denmark

M. Wallentin ( $\square)$

Center of Functionally Integrative Neuroscience,

Aarhus University Hospital, Aarhus, Denmark

e-mail: mikkel@cfin.au.dk

\section{Introduction}

Language can be conceived of as a tool that enables effective and flexible forms of social coordination, such as alignment of perspective (Tylén et al. 2010). Establishing contextual reference during discourse is a vital part of this. Personal pronouns (e.g., he/she/it) are used to refer to recently experienced objects, utterances and events. These items, however, are often no longer present in the environment, and their relations have to be maintained and manipulated in working memory (WM). Spatial relations in cognition are constructed by establishing figure/ground relationships (Talmy 2000), that is, when we see a picture of a man and a woman, we can say that "He is next to her" or that "She is next to him". The difference between these two sentences is that in the first, "he" is the focus of attention and "she" works as the frame of reference for him, and vice versa in the second sentence. Spatial reference frames can originate in the perceiver (in body-, heador eye-centered coordinates) or may refer to absolute coordinates (e.g., north, south), to objects with an intrinsic front/back orientation (e.g., "He is in front of the car" where the car has an intrinsic front) or to a combination of objects and body coordinates (e.g., "He is in front of the tree" where it is the relation between the perceiver and the tree that determines the "in front of" relation) (Levinson 2003). For any given event, there is thus a multitude of possible reference frames, and language can be used to narrow down the scope in order to facilitate exchange of information.

In this paper, we review three studies (Wallentin et al. 2006, 2008a, b, 2011) conducted to investigate the processes underlying the construction of intrinsic reference frames (e.g., "X was in front of $Y$ ") during recall of a previous experiences. It is argued that the dorsal visual 
attention system in the brain, in particular the frontal eye fields, plays a crucial role in this function.

\section{Frontal eye fields (FEFs)}

The frontal eye fields (FEFs) are located in premotor cortex (Blanke et al. 2000). They are known to be involved in eye movement generation, in shifting of spatial attention to present stimuli (Corbetta and Shulman 2002; Greenberg et al. 2010) and during the preparation of anti-saccades, that is, when suppressing the natural tendency to direct gaze toward an appearing target (Munoz and Everling 2004). The FEFs have been found to contain a map of visual space in oculomotor coordinates (Bruce et al. 1985), and lesions to the FEFs cause impairments of voluntary oculomotor control, revealed as an inability to suppress reflexive eye movements toward a distracting stimulus (Milner 1982; Pierrot-Deseilligny et al. 1991).

\section{The "Who is in front of who" image recall task}

In the first functional magnetic resonance imaging (fMRI) experiment (Wallentin et al. 2006, 2008a), participants viewed images depicting a man, a woman and an object (a chair). Subsequently, verbal cues (e.g., "Was he in front of her?"/"Was he older than her?") were used to probe spatial/non-spatial aspects of WM content with an object-relative frame of reference. Egocentric reference frames were evoked by asking additional questions about the relationship between image items and the perceiver (e.g., "Was he in front of you?"). Thus, spatial questions probed either an intrinsic frame of reference or a head-centered, egocentric frame of reference. This task design enabled an investigation of reference frame construction in a naturalistic way during fMRI. The absence of visual stimuli during recall tasks prevented production of actual eye movements that would otherwise introduce confounding activations in the cognitive system of interest. When comparing spatial and non-spatial tasks, a distinct dorsal network of brain regions, including posterior parietal cortex and frontal regions (FEFs), were identified as more active in the spatial conditions (Fig. 1).

\section{The "Who is turned toward who" language recall task}

In a subsequent fMRI study, Wallentin et al. (2008b) examined whether linguistic processing of spatial relations established in a purely linguistic setting (reading and recalling sentences) would call on the same dorsal neural system involved in processing spatial relations set up

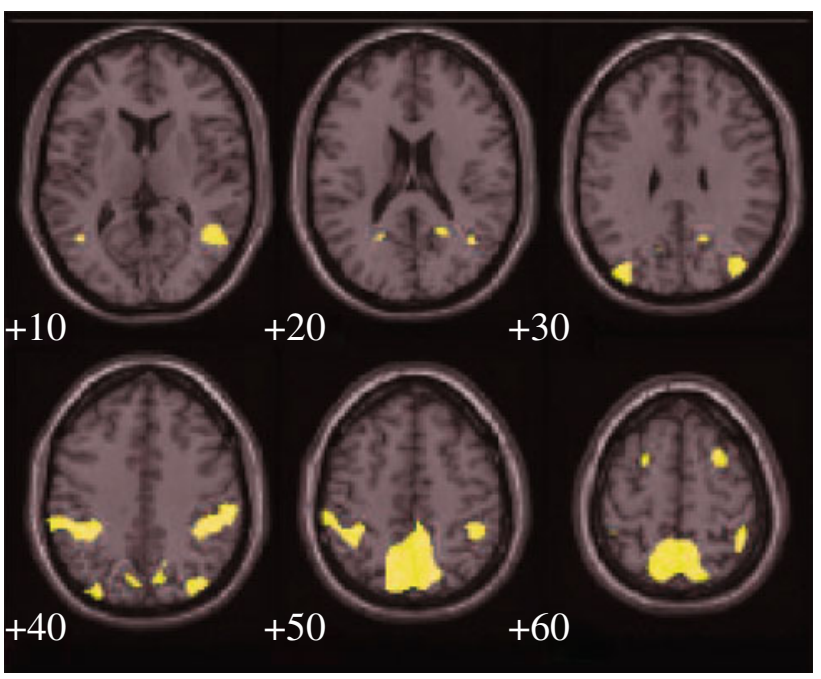

Fig. 1 Image depicting overlap between two fMRI experiments contrasting construction of spatial reference frames to non-spatial reference frames during recall of an image or a sentence. A distinct dorsal network of brain regions was identified, including premotor cortex, putatively the frontal eye fields (adapted from Wallentin et al. Human Brain Mapping, 2008b)

through visual input. Participants in this experiment read simple sentences, describing two persons and their relationship to each other (e.g., "An elderly man is standing behind a young woman in the supermarket cash line"). Similar to the previous experiment, participants were subsequently asked to recall spatial (e.g., "Was he turned toward her?") and equally concrete non-spatial relations (e.g., "Was he older than her?").

Again, we found that recall of the spatial content relative to the non-spatial content resulted in greater activation in the dorsal network of brain regions, including FEFs, strikingly overlapping with the network found to be involved in recall of spatial aspects of images depicting similar scenarios (Fig. 1).

\section{The "Who is in front of who" task revisited}

In a reanalysis of the initial experiment, we investigated correlations between performance on spatial recall and brain responses (Wallentin et al. 2008a). Good performers had both shorter response time and more correct responses than poor performers in all tasks. These behavioral variables were entered into a principal component analysis. The first component reflected generalized performance level. We found that FEFs, bilaterally, had a greater response during recall involving intrinsic frame of reference ("Was he in front of her?") compared with egocentric reference frame ("Was he in front of you") and that this difference was larger in good performers than in poor 
Fig. 2 fMRI contrast between allocentric reference frame construction ("Was he in front of her?") and egocentric reference frame construction ("Was he in front of you?") in FEFs was found to be correlated with overall performance on the task (adapted from Wallentin et al. Neuropsychologia 2008a
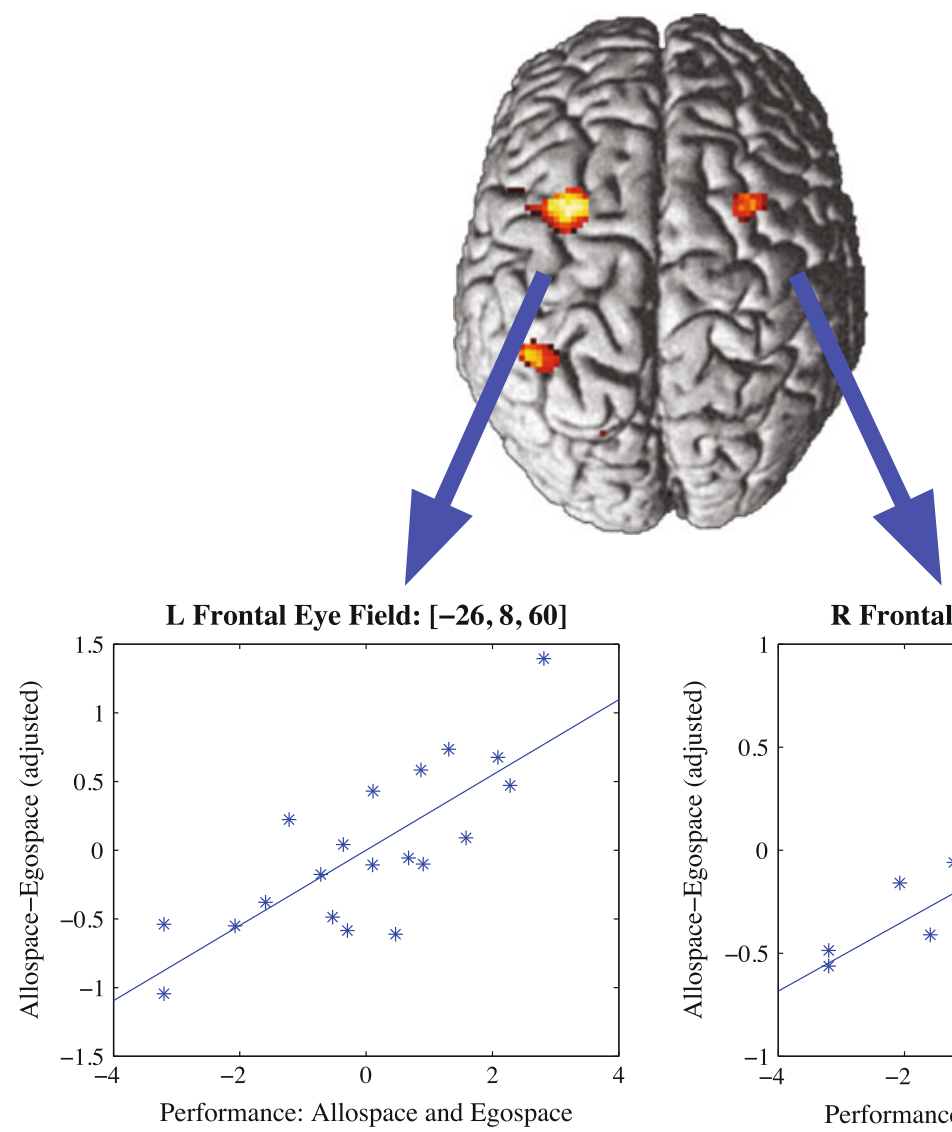

performers (Fig. 2). Our interpretation is that FEFs are involved in simulation of gaze direction shifts during changes of reference frames in representational space.

\section{The mice task}

Blood oxygen level-dependent (BOLD) fMRI, however, is a strictly correlational measure. Additional support for the hypothesis that the FEFs are involved in the construction of spatial reference frames would involve actively interfering with the system. We therefore conducted a dual task interference experiment (Wallentin et al. 2011). Similar to the "Who is in front of who" study, we asked participants to remember both spatial and non-spatial object-centered relations between picture elements and subsequently probed the working memory content by asking questions (e.g., "Was he in front of her?" vs. "Was he darker than her?"). Similar to the previous study, the recall of spatial relations was assumed to involve the FEFs.

In the experiment, participants remembered the configuration of a number of "mice" (see Fig. 3). There were four mice made from simple geometrical shapes (a circle, a square, a triangle and a pentagon), each equipped with eyes and snout to indicate intrinsic orientation. Each mouse was denoted by one of the four Danish third-person personal pronouns. The circle was called "Hun" [she], the square was called "Han" [he], the triangle was called "Den" [it1-third-person common gender], and the pentagon was called "Det" [it2-third-person neuter in Danish]. Participants were trained on these names prior to testing and instructed that their use was merely conventional. The mice differed in luminance (different shades of gray) in order to facilitate non-spatial comparisons (e.g., "Was it darker than her?").

To further manipulate the load on the FEFs, a visual distractor was added, designed to evoke involuntary eye movements. This was displayed coinciding with the recall questions. Studies examining the interplay between voluntary and involuntary saccades have found that an abrupt onset of an irrelevant visual stimulus causes a reliable distraction effect (Irwin et al. 2000; Ludwig and Gilchrist 2002; Schreij et al. 2008; Theeuwes et al. 1999). Visual motion and motion onset, in particular, have also been shown to be a potent capturer of attention in object detection paradigms (Abrams and Christ 2003; Al-Aidroos et al. 2010; Franconeri and Simons 2005; Guo et al. 2010). Based on these findings, we constructed a distractor consisting of an erratically moving dot, appearing and disappearing in the periphery of the screen used for stimulus 


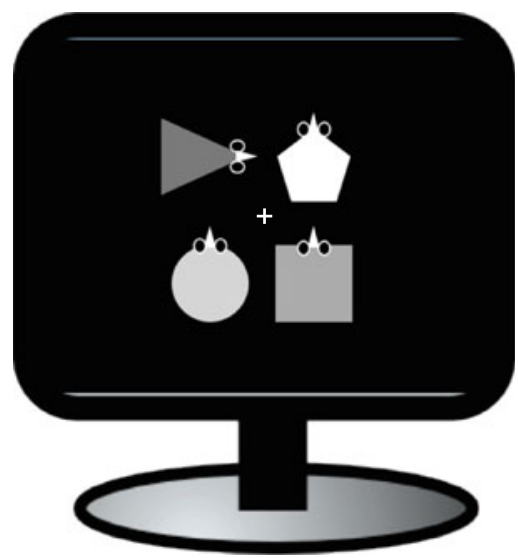

Fig. 3 Left: in the mice task, participants viewed configurations of 2-4 mice (Left) and subsequently recalled either spatial or non-spatial relations in the absence/presence of an eye-motion-evoking visual distractor. Right: response times for spatial (Sp) recall was longer

presentation. Participants were required to ignore the distractor and suppress any urge to move the eyes while solving the mice task. The assumption was that this would force participants to perform suppression of voluntary eye movements, which would depend on the FEFs.

It was found that response times were significantly slower during the spatial recall tasks when the visual distractor was present, whereas the distractor had no effect on the non-spatial task, again suggesting that the eye movement control system is critically involved in constructing spatial reference frames. The distractor had no effect on accuracy, suggesting that interference happens at the level of image reconstruction, not maintenance. Lastly, it was found that the distraction effect was not modulated by task difficulty in terms of the number of remembered items.

\section{Conclusion}

Together, the results from these studies clearly demonstrate a specific role for the brain's eye movement control system (FEFs) in establishing object-relative spatial frame of reference. But while the "in front of" relation with intrinsic frame of reference thus critically involves the eye movement control system, this may not be the whole story for processing of intrinsic frames of reference. Projecting the egocentric notions of "left" and "right" out onto an object has been found to involve an additional body-centered coordinate system (Kessler and Rutherford 2010; Kessler and Thomson 2010; Michelon and Zacks 2006). Future research may shed light on the interaction between these different coordinate systems.

Acknowledgments This paper was supported by the MindLab grant from the Danish Ministry of Science, Technology and Innovation.

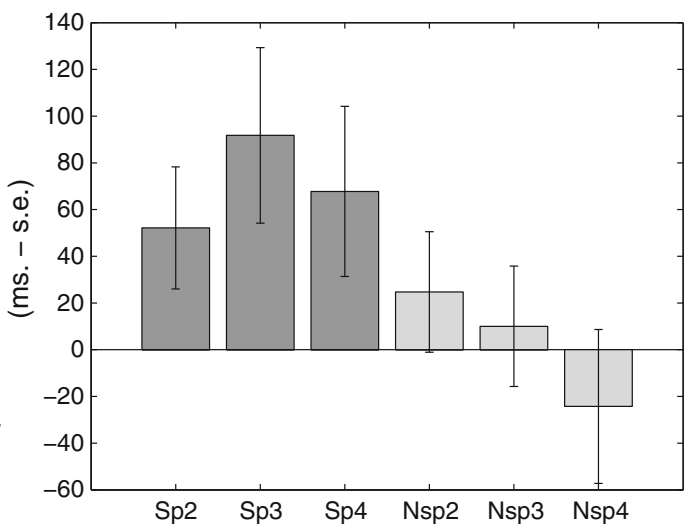

with the distractor, regardless of number of mice, whereas no effect was observed for non-spatial (Nsp) recall (adapted from Wallentin et al. Brain and Cognition 2011)

Conflict of interest This supplement was not sponsored by outside commercial interests. It was funded entirely by ECONA, Via dei Marsi, 78, 00185 Roma, Italy

\section{References}

Abrams RA, Christ SE (2003) Motion onset captures attention. Psychol Sci 14:427-432

Al-Aidroos N, Guo RM, Pratt J (2010) You can't stop new motion: attentional capture despite a control set for colour. Vis Cogn 18:859-880

Blanke O, Spinelli L, Thut G, Michel CM, Perrig S, Landis T, Seeck M (2000) Location of the human frontal eye field as defined by electrical cortical stimulation: anatomical, functional and electrophysiological characteristics. NeuroReport 11:1907-1913

Bruce CJ, Goldberg ME, Bushnell MC, Stanton GB (1985) Primate frontal eye fields. II. Physiological and anatomical correlates of electrically evoked eye movements. J Neurophysiol 54:714-734

Corbetta M, Shulman GL (2002) Control of goal-directed and stimulus-driven attention in the brain. Nat Rev Neurosci 3:215229

Franconeri SL, Simons DJ (2005) The dynamic events that capture visual attention: a reply to Abrams and Christ (2005). Percept Psychophys 67:962-966

Greenberg A, Esterman M, Wilson D, Serences J, Yantis S (2010) Control of spatial and feature-based attention in frontoparietal cortex. J Neurosci 30:14330

Guo RM, Abrams RA, Moscovitch M, Pratt J (2010) Isoluminant motion onset captures attention. Atten Percept Psychophys 72:1311-1316

Irwin DE, Colcombe AM, Kramer AF, Hahn S (2000) Attentional and oculomotor capture by onset, luminance and color singletons. Vis Res 40:1443-1458

Kessler K, Rutherford H (2010) The two forms of visuo-spatial perspective taking are differently embodied and subserve different spatial prepositions. Front Psychol 1:1-12

Kessler K, Thomson LA (2010) The embodied nature of spatial perspective taking: embodied transformation versus sensorimotor interference. Cognition 114:72-88 
Levinson SC (2003) Space in language and cognition. Cambridge University Press, Cambridge

Ludwig CJH, Gilchrist ID (2002) Stimulus-driven and goal-driven control over visual selection. J Exp Psychol Human 28:902-912

Michelon P, Zacks JM (2006) Two kinds of visual perspective taking. Percept Psychophys 68:327-337

Milner B (1982) Some cognitive effects of frontal-lobe lesions in man. Philos Trans R Soc Lond B Biol Sci 298:211-226

Munoz DP, Everling S (2004) Look away: the anti-saccade task and the voluntary control of eye movement. Nat Rev Neurosci 5:218-228

Pierrot-Deseilligny C, Rivaud S, Gaymard B, Agid Y (1991) Cortical control of reflexive visually-guided saccades. Brain $114(\mathrm{Pt}$ 3):1473-1485

Schreij D, Owens C, Theeuwes J (2008) Abrupt onsets capture attention independent of top-down control settings. Percept Psychophys 70:208-218

Talmy L (2000) Toward a cognitive semantics. MIT Press, Cambridge
Theeuwes J, Kramer AF, Hahn S, Irwin DE, Zelinsky GJ (1999) Influence of attentional capture on oculomotor control. J Exp Psychol Human 25:1595-1608

Tylén K, Weed E, Wallentin M, Roepstorff A, Frith CD (2010) Language as a tool for interacting minds. Mind Lang 25:3-29

Wallentin M, Roepstorff A, Glover R, Burgess N (2006) Parallel memory systems for talking about location and age in precuneus, caudate and Broca's region. Neuroimage 32:1850-1864

Wallentin M, Roepstorff A, Burgess N (2008a) Frontal eye fields involved in shifting frame of reference within working memory for scenes. Neuropsychologia 46:399-408

Wallentin M, Weed E, Østergaard L, Mouridsen K, Roepstorff A (2008b) Accessing the mental space-spatial working memory processes for language and vision overlap in precuneus. Hum Brain Mapp 29:524-532

Wallentin M, Kristensen LB, Olsen JH, Nielsen AH (2011) Eye movement suppression interferes with construction of objectcentered spatial reference frames in working memory. Brain Cogn 77:432-437 